\title{
Computational reproducibility
}

Public Domain

\section{Source}

Open Research Glossary

When publishing computational findings, include details and access to the underlying code, data, and implementation. 